\title{
Involvement of miR-30c in resistance to doxorubicin by regulating YWHAZ in breast cancer cells
}

\author{
Y. Fang ${ }^{1}$, H. Shen ${ }^{2}$, Y. Cao ${ }^{1}$, H. Li ${ }^{3}$, R. Qin ${ }^{2}$, Q. Chen ${ }^{1}$, L. Long ${ }^{2}$, X.L. Zhu ${ }^{3}$, C.J. Xie ${ }^{1}$ and W.L. Xu ${ }^{3}$ \\ ${ }^{1}$ Department of Central Laboratory, The First Affiliated People's Hospital, Jiangsu University, Zhenjiang, Jiangsu, China \\ ${ }^{2}$ Department of Oncology, The First Affiliated People's Hospital, Jiangsu University, Zhenjiang, Jiangsu, China \\ ${ }^{3}$ Department of Central Laboratory, The Fourth Affiliated People's Hospital, Jiangsu University, Zhenjiang, Jiangsu, China
}

\begin{abstract}
MicroRNAs (miRNAs) are small RNA molecules that modulate gene expression implicated in cancer, which play crucial roles in diverse biological processes, such as development, differentiation, apoptosis, and proliferation. The aim of this study was to investigate whether miR-30c mediated the resistance of breast cancer cells to the chemotherapeutic agent doxorubicin (ADR) by targeting tyrosine 3-monooxygenase/tryptophan 5-monooxygenase activation protein zeta (YWHAZ). miR-30c was downregulated in the doxorubicin-resistant human breast cancer cell lines MCF-7/ADR and MDA-MB-231/ADR compared with their parental MCF-7 and MDA-MB-231 cell lines, respectively. Furthermore, we observed that transfection of an miR-30c mimic significantly suppressed the ability of MCF-7/ADR to resist doxorubicin. Moreover, the anti-apoptotic gene YWHAZ was confirmed as a target of miR-30c by luciferase reporter assay, and further studies indicated that the mechanism for miR-30c on the sensitivity of breast cancer cells involved YWHAZ and its downstream p38 mitogen-activated protein kinase (p38MAPK) pathway. Together, our findings provided evidence that miR-30c was one of the important miRNAs in doxorubicin resistance by regulating YWHAZ in the breast cancer cell line MCF-7/ADR.
\end{abstract}

Key words: Breast cancer cells; miR-30c; YWHAZ; Doxorubicin resistance

\section{Introduction}

Breast cancer is the most commonly diagnosed cancer worldwide among women and is expected to account for $29 \%$ of all new cancer cases (1). Surgery combined with chemotherapy is the current treatment of choice. However, chemotherapeutic failure frequently contributes to morbidity in patients diagnosed with breast cancer (2), and the acquisition of resistance to doxorubicin is a major clinical obstacle to successful treatment. Consequently, it is essential to elucidate the signaling and regulatory mechanisms that are involved in doxorubicin resistance, which will be helpful for designing new and targeted therapeutic strategies that can overcome drug resistance for the treatment of breast cancer.

MicroRNAs (miRNAs) are a class of small noncoding RNAs, which could bind mRNAs of potentially harmful genes at the $3^{\prime}$-untranslated region (3'-UTR) with perfect or near-perfect complementarity, resulting in degradation or inhibition, respectively, of the target mRNA (3). Generally, one miRNA can modulate hundreds of target genes and one gene can be repressed by hundreds of miRNAs, which results in the formation of complex regulatory pathways. Recently, experimental evidence revealed that miRNAs have been shown to function as regulatory molecules by inhibiting protein translation and to play an important role in development, differentiation, cell proliferation, and apoptosis (3). More recently, some studies suggested that miRNAs are involved in tumor cell resistance and/or sensitivity to chemotherapeutic agents (4). For example, Zhu et al. (5) reported that both miR-451 and miR-27 are involved in the resistance of MCF-7 breast cancer cells to the chemotherapeutic drug doxorubicin mediated by MDR-1 $(5,6)$.

Recent studies suggested that miRNA-30c (miR-30c) is one of the most commonly known tumor suppressor miRNAs, which belongs to the miR-30 family and plays an important role in cell death, invasion, and differentiation (7-9). Bockhorn et al. (10) conducted an miRNA profiling study of clinical breast tumors to show that miR-30c had 
higher expression levels in luminal A tumors and lower levels in claudin-low and basal-like breast tumors, and overexpression of miR-30c could inhibit human breast cancer chemotherapy resistance by regulating twinfilin1 (TWF1) and interleukin-11. Similarly, in this study, we found that the level of miR-30c is lower in doxorubicin (ADR)-resistant human breast cancer cell lines MCF-7/ ADR and MDA-MB-231/ADR compared with their parental cell lines MCF-7 and MDA-MB-231, respectively. Interestingly, our study provided another novel mechanism for understanding doxorubicin resistance, that is, miR-30c could sensitize breast cancer cells to doxorubicin by targeting tyrosine 3-monooxygenase/tryptophan 5-monooxygenase activation protein zeta polypeptide (YWHAZ), a crucial factor in drug resistance.

\section{Material and Methods}

\section{Cell lines and cell culture}

Human breast cancer cell lines MCF-7 and MDA-MB231 were maintained in our laboratory. The breast doxorubicin-resistant cancer cell line MCF-7/ADR was obtained from the Shanghai Institute of Cell Biology (China). Another breast doxorubicin-resistant cancer cell line MDA-MB-231/ADR was established by our laboratory. The method of detecting inducible resistance to doxorubicin was as follows. We continuously added doxorubicin to the growth medium of MDA-MB-231 cells, with a final concentration of $0.1 \mu \mathrm{g} / \mathrm{mL}$. After several passages, clones generated in the presence of doxorubicin were selected as doxorubicin-resistant cancer cells, which had been cultured for more than 6 months in the medium supplemented with doxorubicin to assure that they were truly resistant to doxorubicin. MCF-7 and MDA-MB-231 cell lines were cultured in high-glucose DMEM (Gibco, USA) supplemented with $10 \%$ fetal calf serum (Gibco), and $1 \%$ penicillin and streptomycin (Invitrogen, USA) at $37^{\circ} \mathrm{C}$ in a humidified atmosphere with $5 \% \mathrm{CO}_{2}$. Doxorubicin-resistant cancer cell lines were cultured with $5 \% \mathrm{CO}_{2}$ at $37^{\circ} \mathrm{C}$ in DMEM supplemented with $10 \%$ fetal calf serum, $1 \%$ penicillin and streptomycin, and $1 \mu \mathrm{M}$ insulin. To maintain the ADRresistant phenotype, doxorubicin was added to the culture media at a final concentration of $1 \mu \mathrm{g} / \mathrm{mL}$ MCF-7/ADR and $0.1 \mu \mathrm{g} / \mathrm{mL}$ MDA-MB-231/ADR cells, and they were cultured for 2 weeks in ADR-free medium prior to their use in the experiment.

\section{miRNA transfection}

The miR-30c mimic and its negative control miRNAs were purchased from Shanghai GenePharma Company (China). miR-30c mimic sequences were $3^{\prime}$ CGACUCUCACAUCCUACAAAUGU-5'. We plated cells onto a 6 -well plate at a density of $1 \times 10^{5}$ cells per well $24 \mathrm{~h}$ before transfection with $80 \mathrm{nM}$ miR-30c mimic and its negative control by using Lipofectamine 2000 reagent according to the manufacturer's protocol (Invitrogen). We generally harvested the transfected cells for studies after culturing for $48 \mathrm{~h}$. Three independent experiments were performed.

\section{RNA extraction and quantitative real-time PCR (qRT-PCR) analysis of miRNA}

Small RNAs were extracted from cultured cells by using the RISO ${ }^{\mathrm{TM}}$ RNA ISOlation Reagent (Biomics, USA) following the manufacturer's protocol. qRT-PCR for mature miR-30c was done with the TaqMan stem-loop kit and the TaqMan Universal PCR Master Mix from Applied Biosystems (USA) using the Applied Biosystems AB 7500 real-time PCR system, as described by Chen et al. (11), with U6 small nuclear RNA used as endogenous control for data normalization. The primers for miR-30c were forward: 5'-TGTGTAAACATCCTACACTCTCAG-3' and reverse: 5'-GAGTAAACAACCCTCTCCCA-3'. All reactions were run in triplicate, and miRNA expression was calculated using the $2^{-\Delta \Delta C T}$ method.

\section{qRT-PCR analysis of mRNA expression}

Total RNA was isolated with TRIzol $^{\circledR}$ reagent (Invitrogen) according to the manufacturer's protocol. cDNA was synthesized with the RevertAid First-Strand cDNA Synthesis Kit (Fermentas, USA) according to the manufacturer's instructions. After the reverse transcription reaction, qRT-PCR was conducted in an ABI 7500HT fast real-time PCR System (Applied Biosystems). The relative amount of each mRNA was normalized to $\beta$-actin. Primer sequences used for real-time PCR were as follows: YWHAZ forward and reverse primers were 5' -ATGTACTT GGAAAAAGGCCG-3' and 5'-CCCTGCTCTTGAGGAGC TTA-3', respectively, and $\beta$-actin forward and reverse primers were 5'-GCACCACACCTTCTACAATG-3' and 5'-TGCTTGCTGATCCACATCTG-3', respectively. All real-time PCR assays were conducted in triplicate. The expression levels of each analyte compared with untreated controls was assessed using the $2^{-\Delta \Delta \mathrm{CT}}$ method.

\section{Western blot analysis}

Total cellular extracts were prepared by homogenization of $3 \times 10^{6}$ to $5 \times 10^{6}$ cells in radioimmunoprecipitation assay buffer (Sigma, USA). Equal amounts of proteins $(50 \mu \mathrm{g})$ were separated by SDS-polyacrylamide gel electrophoresis on slab gels of 8 or $12 \%$ polyacrylamide and transferred to nitrocellulose membranes (Millipore Corp., USA). Membranes were incubated with mouse monoclonal antibodies against YWHAZ (1:400, Santa Cruz Biotechnology, USA) and rabbit monoclonal antibodies of phosphorylated ( $p)$-p38 mitogen-activated protein kinase (MAPK) (1:500, Santa Cruz Biotechnology), and $\beta$-actin (1:1000, Cell Signaling, USA) was used as an internal control for protein loading. Antibody binding was revealed by incubation with horseradish peroxidase-conjugated secondary antibodies (Santa Cruz Biotechnology). Signal detection used an enhanced chemiluminescence kit 
(Thermo Scientific, USA) and the ImageJ software system (NIH, USA).

\section{Luciferase reporter assay}

For the luciferase reporter experiments, the $3^{\prime}$-UTR sequence of YWHAZ was amplified by PCR from human genomic DNA using primers that included $X b a l$ and EcoRI tails on the $5^{\prime}$ and $3^{\prime}$ strands, respectively. Primers for YWHAZ 3'-UTR were forward: 5'-CCGCTCGAGCCGGC CTTCCAACCTTTGTC-3' and reverse: 5'-GAATGCGGC CGCTCCCATCATCAATATTTATTGAGCATTTAC-3'. $\mathrm{PCR}$ products were restricted with both $\mathrm{Xbal}$ and $\mathrm{EcoRI}$ restriction endonucleases, and then gel-purified mutations within the putative miR-30c binding sites were performed using a QuikChange site-directed mutagenesis kit (Stratagene, USA). Cells were transfected with the firefly luciferase UTR-report vector, 20 ng control Renilla luciferase pRL-TK vector (Promega, USA), and $10 \mathrm{nM}$ miR30c mimic or a mimic control for the YWHAZ-3'-UTR construct using Lipofectamine 2000 reagent. Forty-eight hours after transfection, cells were lysed with a $1 \times$ passive lysis buffer, and assays were performed using the Dual-Luciferase ${ }^{\mathbb{R}}$ Reporter Assay System kit (Promega) according to the manufacturer's instructions.

\section{Drug sensitivity assay}

Nontransfected or transfected breast cancer cells were seeded onto 96 -well plates with $0.5 \times 10^{4}$ per well in growth medium and incubated at $37^{\circ} \mathrm{C}$ in a humidified $5 \% \mathrm{CO}_{2}$ atmosphere for $48 \mathrm{~h}$, and then treated with doxorubicin (Qilu Pharmaceutical Factory, China) at a concentration range of 0.025 to $0.8 \mu \mathrm{g} / \mathrm{mL}$ for MCF-7 and 0.5 to $16 \mu \mathrm{g} / \mathrm{mL}$ for MCF-7/ADR, respectively. Forty-eight hours after doxorubicin treatment, $10 \mu \mathrm{L}$ CCK-8 solution was added to the medium, and the cells were incubated at $37^{\circ} \mathrm{C}$ for $3 \mathrm{~h}$. The absorbance was read at $570 \mathrm{~nm}$ with a microplate spectrophotometer. Doxorubicin concentrations leading to $50 \%$ cell death $\left(\mathrm{IC}_{50}\right)$ were determined by a CCK-8-dependent cell viability assay. Three independent experiments were performed.

\section{Apoptosis assay}

Doxorubicin was added to the cell medium at a final concentration of $1 \mu \mathrm{M} 48 \mathrm{~h}$ after transfection. After 24-h incubation, cells were collected and assayed with an Annexin V Apoptosis Detection Kit (Beyotime, China) on a BD FACSCalibur ${ }^{\mathrm{TM}}$ System (Becton Dickson, USA) following the manufacturer's instructions. Early apoptotic cells were defined as Annexin-V-positive, propidium iodidenegative cells. Each experiment was performed three times.

\section{Doxorubicin accumulation}

Cells transfected with miR-30c mimic or negative control were treated with $5 \mu \mathrm{M}$ doxorubicin. After 2-h incubation, cells were washed three times with PBS and observed under a fluorescence microscope with a $400 \times$ lens. Quantifications of doxorubicin fluorescence intensity were performed using the ImageJ software.

\section{Small interfering RNA (siRNA) transfection}

SiRNA specific for YWHAZ was chemically synthesized (Guangzhou RuiBio Corp., China) with the following sequence: AGUUCUUGAUCCCCAAUGC-dTdT. Lipofectamine 2000 transfection reagent was mixed with siRNA in a 1:1 ratio (v/v) for $20 \mathrm{~min}$. Cells were incubated with serum-free DMEM $(60 \mathrm{nM})$ as negative control or YWHAZ siRNA for $6 \mathrm{~h}$, and then replaced with regular medium. Forty-eight hours after transfection, the cells were prepared for further analysis. siRNA transfection efficiency was measured with flow cytometry by calculating the percentage of fluorescein-labeled cells. The transfection efficiency was approximately $80 \%$.

\section{MCF-7/ADR-bearing nude mouse model and in vivo treatment}

For preparation of the subcutaneous model, MCF-7/ ADR cells were propagated in 6 -week-old nude mice by weekly transfer of $50 \mu \mathrm{L}$ PBS containing $5 \times 10^{5}$ cells. Mice received a subcutaneous (sc) injection of the cells under the shoulder. After sc implantation of the cells, when the sc tumor was approximately $100 \mathrm{~mm}^{3}$ in size, mice were randomly allocated to groups consisting of five animals each, and $10 \mathrm{nmol}$ miR-30c or mimic negative control in $0.1 \mathrm{~mL}$ saline buffer was locally injected into the tumor mass every 3 days for 2 weeks. Drug administration was given via intraperitoneal injection of $50 \mu \mathrm{L}$ PBS containing $1 \mathrm{mg} / \mathrm{kg}$ doxorubicin, one dose every other day, with three doses total. We monitored tumor growth starting on the first day of treatment and measured the volume of the xenograft every 4 days. Tumor volume (V) was estimated according to the formula: $V=A \times B^{2} / 2 \mathrm{~mm}^{3}$, where $A$ was the largest diameter and $\mathrm{B}$ was the perpendicular diameter. All experiments were performed in accordance with the Guide for the Care and Use of Laboratory Animals (US National Institutes of Health publication \#80-23, revised 1996), with the approval of the Scientific Investigation Board of Jiangsu University, Zhenjiang, China.

\section{Statistical analysis}

All statistical analyses were performed using the SPSS12.0 software (USA). The difference between means was analyzed with the Student $t$-test. $\mathrm{P}$ values of $<0.05$ were considered to be statistically significant. All data are reported as means $\pm S D$.

\section{Results}

\section{Downregulation of miR-30c correlated with overexpression of YWHAZ in breast doxorubicin-resistant cells}

To explore the role of miR-30c in doxorubicin resistance, we first compared miR-30c expression 
between the breast doxorubicin-resistant cancer cells and their parental doxorubicin-sensitive cells. Using miRNA real-time qRT-PCR analysis, we observed a differential expression pattern between them. As shown in Figure 1A, miR-30c expression in MCF-7/ADR was 6.7-fold lower than in MCF-7, and expression was also decreased (1.9fold) in MDA-MB-231/ADR, compared to its parental cell line MDA-MB-231. We subsequently performed a Western blot to detect the expression of YWHAZ. As expected, the expression level of YWHAZ was higher in the doxorubicin-resistant MCF-7/ADR and MDA-MB-231/ ADR cells than that in their parental MCF-7 and MDA-MB231 cells, respectively (Figure $1 \mathrm{~B}$ and $\mathrm{C}$ ). In addition, we detected that the MCF-7 parental and resistant cells showed a more significant difference in the expression of miR-30c, as well as YWHAZ. These results potentially indicated that loss of miR-30c might be associated with overexpression of YWHAZ in breast doxorubicin-resistant cells.

A

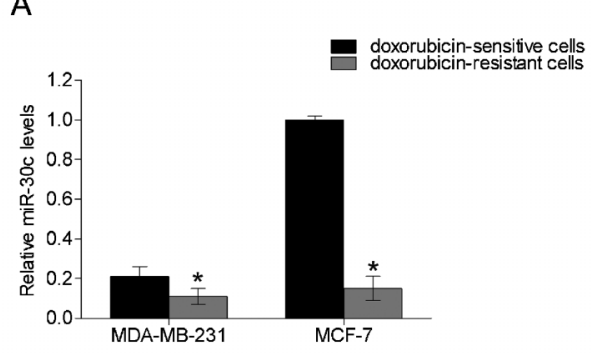

B

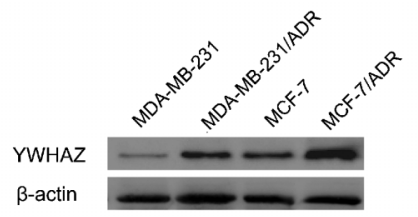

C

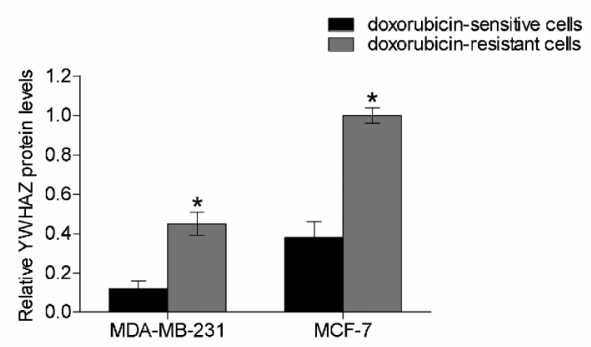

Figure 1. Down-regulation of miR-30c correlated with overexpression of YWHAZ in breast doxorubicin-resistance cells. $A$, qRT-PCR analysis of miR-30c expression in breast doxorubicin (ADR)-resistant and doxorubicin-sensitive cells. Data are shown as fold-changes of miRNA levels in MCF-7/ADR cell line relative to MCF-7 cell line, or in MDA-MB-231/ADR cell line relative to MDA-MB-231. ${ }^{*} \mathrm{P}<0.05$, Student $t$-test. $B$ and $C$, Expression levels of YWHAZ in breast doxorubicin-sensitive and doxorubicinresistant cell lines. ${ }^{*} \mathrm{P}<0.05$, Student $t$-test.

\section{Overexpression of $\mathrm{miR}-30 \mathrm{c}$ sensitized MCF-7/ADR cells to doxorubicin}

In the next series of studies, we established the relationship between miR-30c expression and doxorubicin resistance using MCF-7/ADR and its parental cell line MCF-7 because of the significant difference in miR-30c expression. As shown in Figure 2A, the level of drug resistance between the parent and resistant cells was 36.5 times $\left(\mathrm{IC}_{50}\right.$ was $0.331 \pm 0.121 \mu \mathrm{g} / \mathrm{mL}$ for MCF-7 and $12.078 \pm 0.368 \mu \mathrm{g} / \mathrm{mL}$ for MCF-7/ADR, respectively, $P<0.05)$. To investigate whether miR-30c modulated chemosensitivity in breast cancer, we transfected $80 \mathrm{nM}$ miR-30c mimic or negative control into MCF-7/ADR cells. Stem-loop real-time PCR revealed that the miR30c mimic significantly increased miR-30c expression, suggesting that miR-30c was efficiently transfected into cells (Figure 2B). In the CCK-8 assay, MCF-7/ADR cells transfected with miR-30c mimic exhibited greatly enhanced sensitivity to doxorubicin, compared with those transfected with the control miRNA mimic $\left(\mathrm{IC}_{50}\right.$ was $4.008 \pm 0.342$ and $10.977 \pm 0.275 \mu \mathrm{g} / \mathrm{mL}$, respectively; $\mathrm{P}<0.05$; Figure $2 \mathrm{C}$ ), suggesting the role of miR$30 \mathrm{c}$ in mediating sensitivity to doxorubicin in breast cancer cells. In order to strengthen this argument, we observed the intracellular accumulation of doxorubicin in MCF-7/ADR cells under a fluorescence microscope. As shown in Figure 2D, intracellular accumulation of doxorubicin was increased in cells treated with miR-30c mimic in comparison with control cells. Furthermore, we examined the effect of miR-30c overexpression on apoptotic cell death. A flow cytometry assay for miR$30 \mathrm{c}$ verified that breast cancer cells transfected with miR-30c mimic had a significantly higher apoptosis rate than the negative control group induced by doxorubicin $(P<0.01$; Figure 2E). These results demonstrated that miR-30c restoration sensitizes MCF-7/ADR cells to doxorubicin.

miR-30c suppressed expression of the YWHAZ gene

To explore the downstream mechanism through which miR-30c modulates doxorubicin resistance, we first used the algorithms for target gene prediction, including TargetScan and miRanda, and found that YWHZA was the potential target gene of miR-30c (Figure 3A). To further detect whether YWHAZ is the target gene of miR-30c, we cotransfected miR-30c mimic or the negative control together with the luciferase reporter plasmid with the $3^{\prime}$-UTR of YWHAZ into MCF-7/ ADR cells. As shown in Figure 3B, reporter activity of the $3^{\prime}$-UTR of YWHAZ was significantly suppressed by miR30c mimic, whereas mutant YWHAZ $3^{\prime}$-UTR-luciferase activity remained unchanged in cells transfected with miR-30c mimic. These results indicated that YWHAZ was the target gene of miR-30c. We consequently conducted further analysis to explore whether miR-30c regulated YWHAZ expression in MCF-7/ADR cells. Results showed 
A

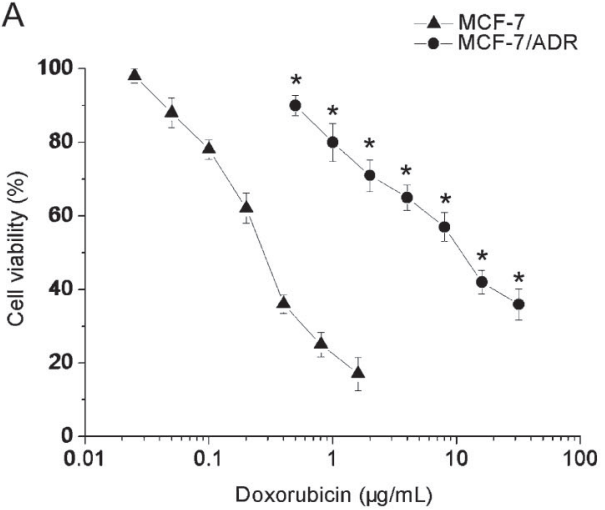

C

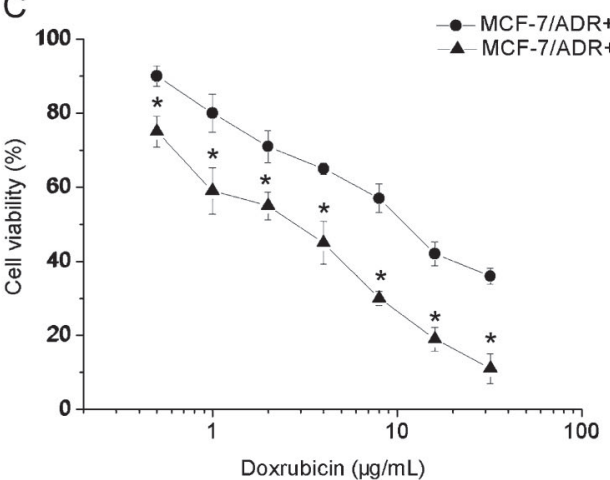

B

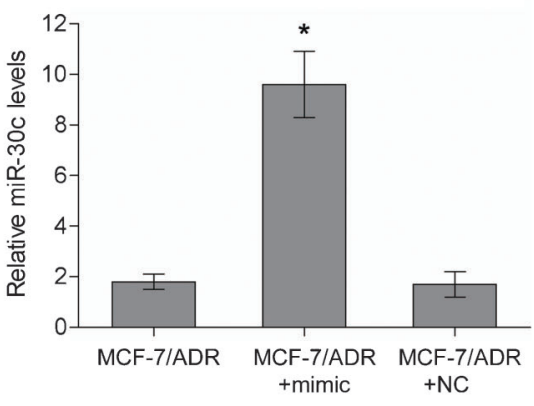

D

MCF-7/ADR+NC

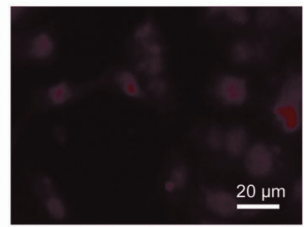

MCF-7/ADR+mimic

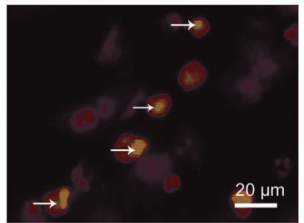

$E$

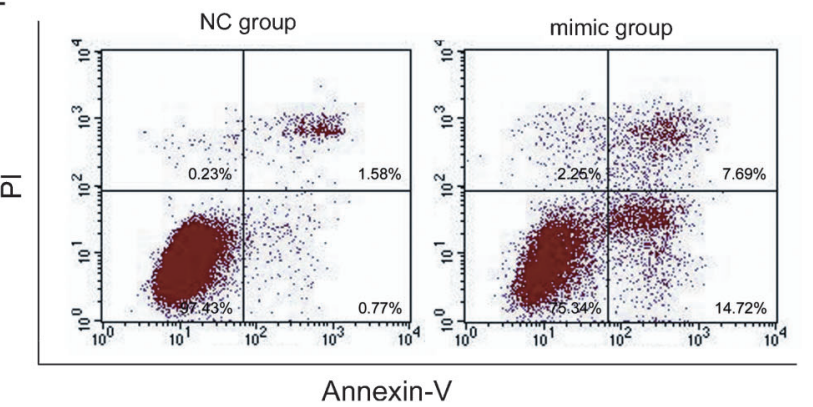

Figure 2. Overexpression of miR-30c sensitized the MCF-7/ADR cells to doxorubicin (ADR). A, Survival curves of MCF-7/ADR and MCF-7 cells. The cells were treated with various doses of doxorubicin. After 48-h incubation, the viability of cells was measured with the CCK-8 assay. The MCF-7/ADR cell line showed a 36.5 -fold acquired resistance to doxorubicin based on $\mathrm{IC}_{50}(12.078 \pm 0.368 \mathrm{vs} 0.331 \pm 0.121 \mu \mathrm{g} /$ $\mathrm{mL}$; ${ }^{*} \mathrm{P}<0.05$, Student $t$-test). $B$, qRT-PCR showed that the expression of miR-30c significantly increased in the cells transfected with miR30c mimic ( ${ }^{*} \mathrm{P}<0.05$, Student $t$-test). $C$, After transfection with miR-30c mimic or negative control (NC) for $48 \mathrm{~h}, \mathrm{MCF}-7 / \mathrm{ADR}$ cells were subsequently treated with various doses of doxorubicin for $48 \mathrm{~h}$. Cell viability was determined using the CCK-8 assay. The IC 50 of miR-30c mimic and NC groups was $4.008 \pm 0.342$ and $10.977 \pm 0.275 \mu \mathrm{g} / \mathrm{mL}\left({ }^{*} \mathrm{P}<0.05\right.$, Student $t$-test). $D$, After incubation with $5 \mu \mathrm{M}$ doxorubicin for $2 \mathrm{~h}$, the cells were washed three times with PBS, and then observed under a fluorescence microscope with a $400 \times$ lens. The intracellular accumulation of doxorubicin was more significant in the mimic group as indicated by arrows. E, Overexpression of miR-30c in MCF-7/ADR cells rescued doxorubicin-induced apoptosis after $48 \mathrm{~h}$ of $1 \mu \mathrm{M}$ doxorubicin treatment.

that there was a consistent and strong inverse correlation between miR-30c levels and YWHAZ. Transfected miR-30c mimic downregulated YWHAZ expression at mRNA and protein levels in MCF-7/ADR cells (Figure 3C and D).
YWHAZ was a key signal molecule in doxorubicin resistance by reducing activation of the p38MAPK signal pathway in MCF-7/ADR cells

Previous studies have shown that YWHAZ is involved in drug resistance in breast cancer cells (12). However, its 
A

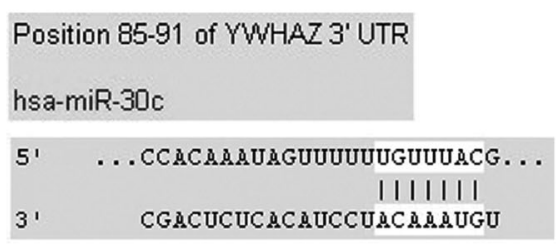

C

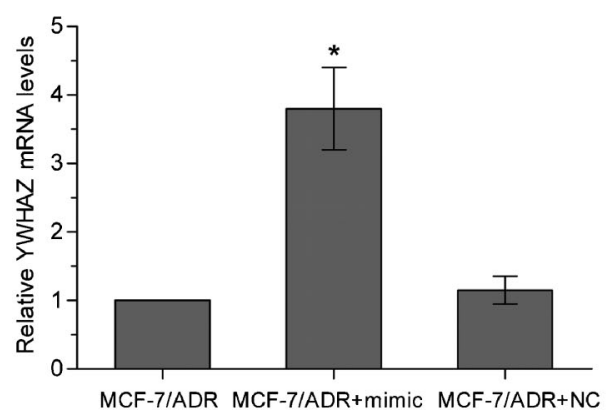

B

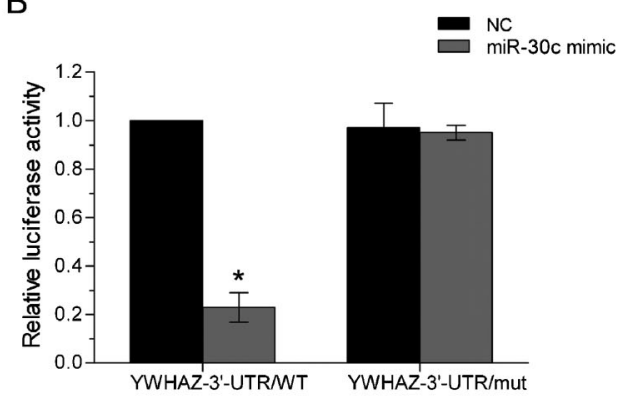

D

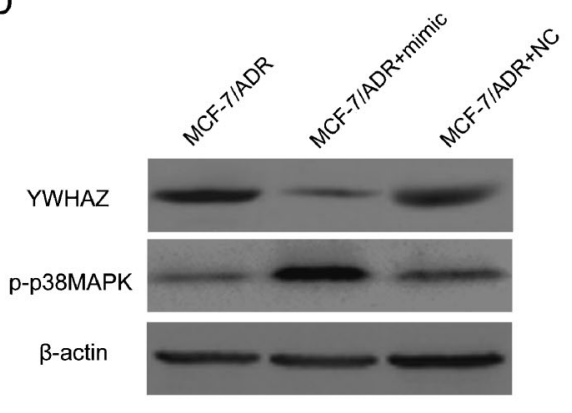

Figure 3. miR-30c suppressed the expression of the YWHAZ gene. A, Sequence alignment between miR-30c and the target $Y W H A Z$ $3^{\prime}$-UTR. B, Effect of miR-30c on YWHAZ was assessed with the luciferase reporter system. The miR-30c mimic, together with the luciferase reporter vector or control vector, was co-transfected into the MCF-7/ADR cells $\left({ }^{*} \mathrm{P}<0.05\right.$, Student $t$-test). $C$, qRT-PCR measured the levels of YWHAZ mRNA in the MCF-7/ADR cells and MCF-7/ADR cells transfected with miR-30c mimic or negative control ( ${ }^{*} \mathrm{P}<0.05$, Student $t$-test). $D$, Western blot analysis of $\mathrm{YWHAZ}$ and $\mathrm{p}$-p38MAPK protein expression in the MCF-7/ADR cells and MCF-7/ADR cells after transfection with miR-30c mimic or negative control. ADR: doxorubicin; NC: negative control.

role in doxorubicin resistance in MCF-7/ADR cells remains unclear. To determine the impact of $Y W H A Z$ alone on cell viability, we transfected SiRNA against YWHAZ or a negative control into MCF-7/ADR cells, followed by treatment with various doses of doxorubicin. The results suggested that $Y W H A Z$ siRNA effectively reduced protein levels of YWHAZ (Figure 4A and B), and, as shown in Figure 4D, the MCF-7/ADR cells that were transfected with YWHAZ siRNA had a lower survival rate than the negative control group $\left(\mathrm{IC}_{50}\right.$ was $3.125 \pm 0.401 \mu \mathrm{g} / \mathrm{mL}$ for the siYWHAZ group and $10.178 \pm 0.344 \mu \mathrm{g} / \mathrm{mL}$ for the negative control group, respectively, $\mathrm{P}<0.05)$. Moreover, the survival pattern of MCF-7/ADR cells transfected with YWHAZ siRNA was similar to that of cells with miR-30c overexpression. For the above-mentioned results, we suggested that miR-30c modulated doxorubicin resistance in MCF-7/ADR cells by downregulating YWHAZ. Notably, it has been reported that the activated p38MAPK pathway plays a causal role in chemotherapy-induced apoptosis in resistant cells $(13,14)$. It has also been reported that enhanced chemosensitivity by suppression of YWHAZ seems to be associated with activation of the p38MAPK pathway (15). Therefore, we examined the impact of miR$30 \mathrm{c}$ on the p38MAPK pathway and found that the level of
p38MAPK phosphorylation was increased, along with the reduction of YWHAZ expression levels (Figure 3D). We also observed that the level of p38MAPK phosphorylation in siYWHAZ-transfected cells was increased compared to controls and scrambled siRNA-transfected cells (Figure 4A and $\mathrm{C}$ ). Therefore, we concluded that overexpression of miR-30c may activate the p38MAPK pathway by reducing protein levels of YWHAZ to enhance doxorubicin sensitivity in MCF-7/ADR cells; however, the mechanism remains to be further studied.

\section{miR-30c regulated doxorubicin resistance in vivo}

Nude mice with doxorubicin-resistant human breast cancer and Sc passage of MCF-7/ADR were used to validate the role of miR-30c as a regulator of breast tumor progression in vivo. As shown in Figure 5A, the tumor size of the miR-30c mimic group was significantly smaller than that of the negative control group. Tumor growth was much slower for the group given doxorubicin and miR-30c mimic compared with the negative control group (Figure $5 \mathrm{~B}$ ). Taken together, at a dose of $1 \mathrm{mg} / \mathrm{kg}$, doxorubicin did not inhibit tumor growth in the negative control group, but significantly inhibited the growth of tumor cells in the miR$30 \mathrm{c}$ overexpression group. We, therefore, identified miR-30c 
A

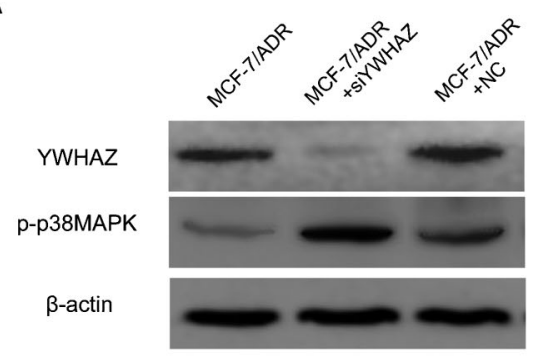

C

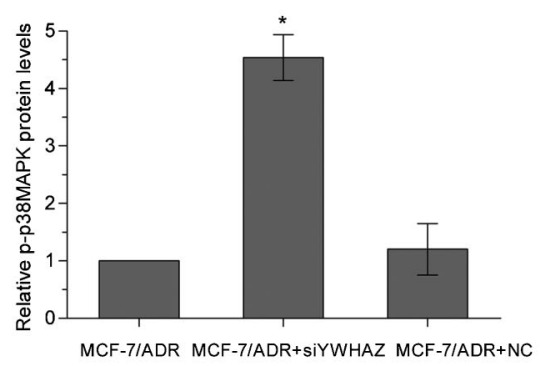

B

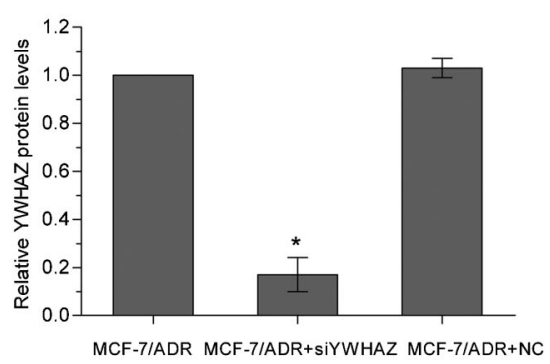

D

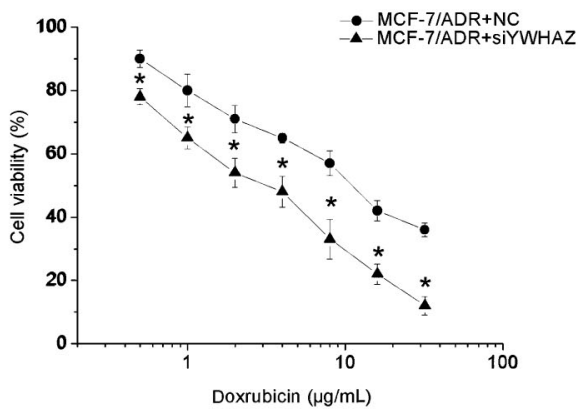

Figure 4. YWHAZ was a key signal molecule in doxorubicin (ADR) resistance by reducing the activation of p38MAPK signal pathway in MCF-7/ADR cells. $A, B$ and $C$, YWHAZ and p-p38MAPK protein levels in MCF-7/ADR cells transfected with YWHAZ small interfering (si) RNA or a scrambled siRNA ( ${ }^{*} \mathrm{P}<0.05$, Student $t$-test). $D$, After 48 -h transfection, MCF-7/ADR cells were treated with various doses of doxorubicin. Cell viability was determined using the CCK-8 assay ( ${ }^{*} \mathrm{P}<0.05$, Student $t$-test). NC: negative control.

as playing a key role in reversing resistance of the MCF-7/ ADR-bearing nude mice model to doxorubicin treatment in vivo.

\section{Discussion}

Breast cancer is a heterogeneous disease due to a complicated etiology involving both genetic and environmental factors. Although advances in both diagnosis and treatment, which incorporates surgery, radiation therapy, and chemotherapy, have led to the improvement of prognosis $(16,17)$, chemotherapy resistance remains a major challenge in breast cancer therapy. Previous studies have shown that miRNA could serve as an "oncogene" or "tumor suppressor gene" and regulate different cellular processes by targeting hundreds of genes (18-22). Functional studies indicate that miRNAs participate in regulating almost every cellular process, including drug resistance. However, the role of miRNA in drug resistance in tumor cells remains largely unexplored. In this report, the molecular mechanism involved in miR$30 \mathrm{c}$ and its specific target YWHAZ in MCF-7/ADR provided new evidence for the development of drug resistance in cancer cells.

Recently, an increasing number of studies have focused on the anticancer properties of miR-30c, showing deregulation of miR-30c expression in a variety of human cancers (23-26). For example, Rodriguez-Gonzalez et al. (27) reported miRNA-30c as an independent predictor of the clinical benefit of tamoxifen therapy in patients with advanced breast cancer. Mu and Su (28) showed that the single nucleotide polymorphism A/G in pre-miR-30c might be associated with a high risk of gastric cancer. miRNA has also been shown to potentially act as a tumor suppressor in these tumors. However, the biological relationship between miR-30c and its specific target YWHAZ in doxorubicin resistance in MCF-7/ADR cells remains unknown.

Targeting at the apoptotic pathway could be an attractive and therapeutically beneficial option because chemotherapeutically induced cell death is generally programmed by apoptosis (29). YWHAZ codes for a known anti-apoptotic protein, $14-3-3 \zeta$, which is a member of the highly conserved family of seven 14-3-3 proteins, all encoded by different genes (30). YWHAZ serves as a pivotal factor that binds and stabilizes key proteins involved in signal transduction, cell proliferation, and apoptosis, including EGFR, HER2, PKC, $\beta$-catenin, and RAF-1 (31-34). Additionally, overexpression of YWHAZ contributed to de novo chemoresistance to anthracyclines and is permissive for metastatic recurrence (12). Moreover, p38MAPK signaling is well known as an independent parallel MAPK pathway that is believed to play an important role in apoptosis, and the activation of 
A

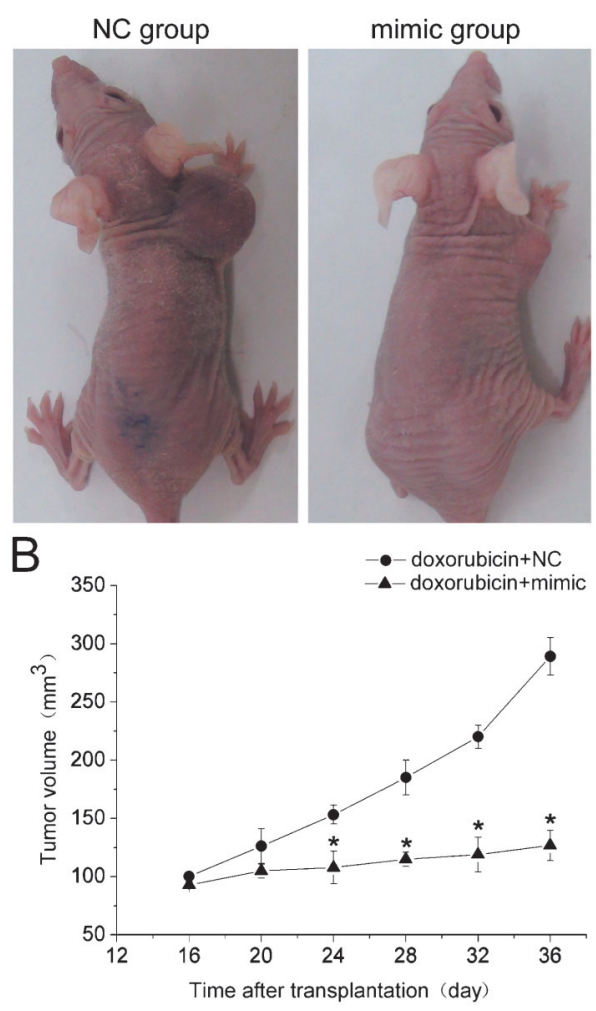

Figure 5. miR-30c regulated doxorubicin (ADR) resistance in vivo. The experiment was carried out using 6-week-old nude mice implanted subcutaneously (sc) with $5 \times 10^{5}$ MCF-7/ADR cells weekly. The treatments were administered as indicated in Material and Methods. A, The nude mice bearing tumors at 36 days after the $s c$ transplantation are shown. NC: negative control. $B$, Curves of tumor growth in the nude xenografts of the MCF-7/ ADR cells ( ${ }^{*} \mathrm{P}<0.05$, Student $t$-test).

p38MAPK phosphorylation reverses resistance to apoptosis in different types of tumors, including breast carcinoma $(35,36)$. Furthermore, the anti-apoptotic gene YWHAZ specifically regulates the p38MAPK signaling pathway, playing a key role in tumor formation and drug resistance $(15,37)$. Together, we can hypothesize that overexpression of miR-30c may result in reduced expression of YWHAZ as well as its downstream p38MAPK pathway, and, therefore, may reverse doxorubicin resistance in MCF-7/ADR cells.

\section{References}

1. Siegel R, Naishadham D, Jemal A. Cancer statistics, 2012. CA Cancer J Clin 2012; 62: 10-29, doi: 10.3322/caac. 20138.

2. Sorlie T, Perou CM, Fan C, Geisler S, Aas T, Nobel A, et al. Gene expression profiles do not consistently predict the clinical treatment response in locally advanced breast
To confirm this hypothesis, we first examined the expression of miR-30c and found that the level of expression was significantly decreased in doxorubicinresistant cell lines MCF-7/ADR and MDA-MB-231/ADR, compared with their corresponding parental cell lines MCF-7 and MDA-MB-231, respectively, which indicated that reduced miR-30c levels may be associated with doxorubicin resistance in breast cancer. To further investigate the function of miR-30c, we predicted binding sites for miR-30c in the YWHAZ 3'-UTR by bioinformatic analysis. Western blot assay indicated that increased expression of miR-30c might have an impact on YWHAZ expression. Furthermore, we demonstrated that YWHAZ was a target gene of miR-30c by luciferase reporter assay. Then, we transfected the chemically synthesized miR-30c mimic oligonucleotides or YWHAZ siRNA into MCF-7/ADR cells. Results showed that restoration of miR-30c or inhibition of YWHAZ in MCF-7/ADR cells sensitized MCF-7/ADR cells to doxorubicin. Importantly, experiments in vivo showed that doxorubicin significantly inhibited the growth of tumor cells in the miR-30c overexpression group, but not in the negative control group, which strongly confirmed our argument. Consistent with these findings, we also found that overexpression of miR-30c led to downregulation of YWHAZ and a more active signaling through the p38MAPK pathway, which contributed to reversing doxorubicin resistance in MCF-7/ ADR cells.

In conclusion, we provided direct evidence that miR30c restoration sensitizes MCF-7/ADR to doxorubicin treatment in vivo and in vitro. Furthermore, we documented miR-30c as a potential tumor suppressor of the progression to doxorubicin resistance in breast cancer through its ability to target YWHAZ and the p38MAPK signaling pathway, providing new mechanistic insights into the function of miRNA as a potential therapy for a variety of cancers. Finally, the therapeutic modulation of miR-30c and other miRNAs might be worth exploring in an attempt to improve drug efficacy by overcoming mechanisms of resistance.

\section{Acknowledgments}

Research supported by grants from the National Natural Science Foundation of China (\#81070423 and \#81101677) and the Natural Science Foundation of Jiangsu Province of China (\#BK2010332).

cancer. Mol Cancer Ther 2006; 5: 2914-2918, doi: 10.1158/1535-7163.MCT-06-0126.

3. Bartel DP. MicroRNAs: genomics, biogenesis, mechanism, and function. Cell 2004; 116: 281-297, doi: 10.1016/S00928674(04)00045-5.

4. Blower PE, Chung JH, Verducci JS, Lin S, Park JK, Dai Z, 
et al. MicroRNAs modulate the chemosensitivity of tumor cells. Mol Cancer Ther 2008; 7: 1-9, doi: 10.1158/15357163.MCT-07-0573.

5. Zhu H, Wu H, Liu X, Evans BR, Medina DJ, Liu CG, et al. Role of MicroRNA miR-27a and miR-451 in the regulation of MDR1/P-glycoprotein expression in human cancer cells. Biochem Pharmacol 2008; 76: 582-588, doi: 10.1016/j.bcp. 2008.06.007.

6. Kovalchuk O, Filkowski J, Meservy J, Innytskyy Y, Tryndyak VP, Chekhun VF, et al. Involvement of microRNA-451 in resistance of the MCF-7 breast cancer cells to chemotherapeutic drug doxorubicin. Mol Cancer Ther 2008; 7: 21522159, doi: 10.1158/1535-7163.MCT-08-0021.

7. Poudel S, Song J, Jin EJ, Song K. Sulfuretin-induced miR$30 \mathrm{C}$ selectively downregulates cyclin D1 and D2 and triggers cell death in human cancer cell lines. Biochem Biophys Res Commun 2013; 431: 572-578, doi: 10.1016/ j.bbrc.2013.01.012.

8. Bockhorn J, Yee K, Chang YF, Prat A, Huo D, Nwachukwu $\mathrm{C}$, et al. MicroRNA-30c targets cytoskeleton genes involved in breast cancer cell invasion. Breast Cancer Res Treat 2013; 137: 373-382, doi: 10.1007/s10549-012-2346-4.

9. Wu T, Zhou H, Hong $Y$, Li J, Jiang $X$, Huang H. miR-30 family members negatively regulate osteoblast differentiation. J Biol Chem 2012; 287: 7503-7511, doi: 10.1074/jbc. M111.292722.

10. Bockhorn J, Dalton R, Nwachukwu C, Huang S, Prat A, Yee $\mathrm{K}$, et al. MicroRNA-30c inhibits human breast tumour chemotherapy resistance by regulating TWF1 and IL-11. Nat Commun 2013; 4: 1393, doi: 10.1038/ncomms2393.

11. Chen C, Ridzon DA, Broomer AJ, Zhou Z, Lee DH, Nguyen JT, et al. Real-time quantification of microRNAs by stemloop RT-PCR. Nucleic Acids Res 2005; 33: e179, doi: 10.1093/nar/gni178.

12. Li Y, Zou L, Li Q, Haibe-Kains B, Tian R, Li Y, et al. Amplification of LAPTM4B and YWHAZ contributes to chemotherapy resistance and recurrence of breast cancer. Nat Med 2010; 16: 214-218, doi: 10.1038/nm.2090.

13. Brozovic A, Fritz G, Christmann M, Zisowsky J, Jaehde U, Osmak $\mathrm{M}$, et al. Long-term activation of SAPK/JNK, p38 kinase and fas- $\mathrm{L}$ expression by cisplatin is attenuated in human carcinoma cells that acquired drug resistance. Int $J$ Cancer 2004; 112: 974-985, doi: 10.1002/ijc.20522.

14. Zhang $Y$, Huang $Y$, Rishi AK, Sheikh MS, Shroot B, Reichert $\mathrm{U}$, et al. Activation of the p38 and JNK/SAPK mitogenactivated protein kinase pathways during apoptosis is mediated by a novel retinoid. Exp Cell Res 1999; 247: 233-240, doi: 10.1006/excr.1998.4350.

15. Choi JE, Hur W, Jung CK, Piao LS, Lyoo K, Hong SW, et al. Silencing of 14-3-3zeta over-expression in hepatocellular carcinoma inhibits tumor growth and enhances chemosensitivity to cis-diammined dichloridoplatium. Cancer Lett 2011; 303: 99-107, doi: 10.1016/j.canlet.2011.01.015.

16. Naumov GN, Townson JL, MacDonald IC, Wilson SM, Bramwell VH, Groom AC, et al. Ineffectiveness of doxorubicin treatment on solitary dormant mammary carcinoma cells or late-developing metastases. Breast Cancer Res Treat 2003; 82: 199-206, doi: 10.1023/B:BREA.0000004377.12288.3c.

17. Rivera E, Gomez H. Chemotherapy resistance in metastatic breast cancer: the evolving role of ixabepilone. Breast Cancer Res 2010; 12 (Suppl 2): S2, doi: 10.1186/bcr2573.
18. Lee $\mathrm{CH}$, Kuo $\mathrm{WH}$, Lin $\mathrm{CC}$, Oyang $\mathrm{YJ}$, Huang $\mathrm{HC}$, Juan HF. MicroRNA-regulated protein-protein interaction networks and their functions in breast cancer. Int J Mol Sci 2013; 14: 11560-11606, doi: 10.3390/ijms140611560.

19. Gao J, Li L, Wu M, Liu M, Xie X, Guo J, et al. MiR-26a inhibits proliferation and migration of breast cancer through repression of MCL-1. PLoS One 2013; 8: e65138, doi: 10.1371/journal.pone.0065138.

20. Yang G, Wu D, Zhu J, Jiang O, Shi Q, Tian J, et al Upregulation of miR-195 increases the sensitivity of breast cancer cells to Adriamycin treatment through inhibition of Raf-1. Oncol Rep 2013; 30: 877-889.

21. Bisso A, Faleschini M, Zampa F, Capaci V, De Santa J, Santarpia L, et al. Oncogenic miR-181a/b affect the DNA damage response in aggressive breast cancer. Cell Cycle 2013; 12: 1679-1687, doi: 10.4161/cc.24757.

22. Nassirpour R, Mehta PP, Baxi SM, Yin MJ. miR-221 promotes tumorigenesis in human triple negative breast cancer cells. PLoS One 2013; 8: e62170, doi: 10.1371/ journal.pone.0062170.

23. Garofalo M, Romano G, Di Leva G, Nuovo G, Jeon YJ, Ngankeu A, et al. EGFR and MET receptor tyrosine kinasealtered microRNA expression induces tumorigenesis and gefitinib resistance in lung cancers. Nat Med 2012; 18: 7482, doi: $10.1038 / \mathrm{nm} .2577$

24. Li XH, Ha CT, Fu D, Xiao M. Micro-RNA30c negatively regulates REDD1 expression in human hematopoietic and osteoblast cells after gamma-irradiation. PLoS One 2012; 7: e48700, doi: 10.1371/journal.pone.0048700.

25. Marchand A, Proust C, Morange PE, Lompre AM, Tregouet DA. miR-421 and miR-30c inhibit SERPINE 1 gene expression in human endothelial cells. PLoS One 2012; 7 : e44532, doi: 10.1371/journal.pone.0044532.

26. Chen JP, Liu Y, Hu ZB, Shen HB. [Single nucleotide polymorphism in flanking region of miR-30c influences the maturing process of miR-30c in lung carcinoma]. Zhonghua Zhong Liu Za Zhi 2012; 34: 664-668.

27. Rodriguez-Gonzalez FG, Sieuwerts AM, Smid M, Look MP, Meijer-van Gelder ME, de Weerd V, et al. MicroRNA-30c expression level is an independent predictor of clinical benefit of endocrine therapy in advanced estrogen receptor positive breast cancer. Breast Cancer Res Treat 2011; 127 : 43-51, doi: 10.1007/s10549-010-0940-x.

28. Mu YP, Su XL. Polymorphism in pre-miR-30c contributes to gastric cancer risk in a Chinese population. Med Oncol 2012; 29: 1723-1732, doi: 10.1007/s12032-011-0115-6.

29. Baguley BC. Tumor stem cell niches: a new functional framework for the action of anticancer drugs. Recent Pat Anticancer Drug Discov 2006; 1: 121-127, doi: 10.2174/ 157489206775246494.

30. Tzivion G, Gupta VS, Kaplun L, Balan V. 14-3-3 Proteins as potential oncogenes. Semin Cancer Biol 2006; 16: 203-213, doi: 10.1016/j.semcancer.2006.03.004.

31. Ando K, Ozaki T, Yamamoto H, Furuya K, Hosoda M, Hayashi $\mathrm{S}$, et al. Polo-like kinase 1 (Plk1) inhibits p53 function by physical interaction and phosphorylation. J Biol Chem 2004; 279: 25549-25561, doi: 10.1074/jbc.M314182200.

32. Costa RH. FoxM1 dances with mitosis. Nat Cell Biol 2005; 7: 108-110, doi: 10.1038/ncb0205-108.

33. Filipowicz W, Bhattacharyya SN, Sonenberg N Mechanisms of post-transcriptional regulation by 
microRNAs: are the answers in sight? Nat Rev Genet 2008; 9: 102-114, doi: 10.1038/nrg2290.

34. McPherson RA, Harding A, Roy S, Lane A, Hancock JF. Interactions of C-Raf-1 with phosphatidylserine and 14-3-3. Oncogene 1999; 18: 3862-3869, doi: 10.1038/sj.onc. 1202730.

35. Mansouri A, Ridgway LD, Korapati AL, Zhang Q, Tian L, Wang $Y$, et al. Sustained activation of JNK/p38 MAPK pathways in response to cisplatin leads to Fas ligand induction and cell death in ovarian carcinoma cells. $J$ Biol Chem 2003; 278: 19245-19256, doi: 10.1074/jbc.
M208134200.

36. Weir NM, Selvendiran K, Kutala VK, Tong L, Vishwanath S, Rajaram M, et al. Curcumin induces G2/M arrest and apoptosis in cisplatin-resistant human ovarian cancer cells by modulating Akt and p38 MAPK. Cancer Biol Ther 2007; 6: 178-184, doi: 10.4161/cbt.6.2.3577.

37. Min S, Liang X, Zhang M, Zhang Y, Mei S, Liu J, et al. Multiple tumor-associated microRNAs modulate the survival and longevity of dendritic cells by targeting YWHAZ and Bcl2 signaling pathways. J Immunol 2013; 190: 2437-2446, doi: 10.4049/jimmunol.1202282. 